\title{
Method and results of studying the influence of grinder knife parameters on specific work of feed cutting
}

\author{
Nikolay Ayugin*, Vitaly Isaychev, Rustam Khalimov, Nikolay Semashkin \\ Ulyanovsk State Agrarian University named after P.A. Stolypin, 432017 Ulyanovsk, Russia
}

\begin{abstract}
To study the process of feed cutting of vegetable origin, a laboratory setup based on the principle of an impact machine, which made it possible to provide a cutting speed in a sufficiently wide range, was developed at Ulyanovsk State Agrarian University. To ensure the required sliding angle, the cutting element (knife) has an adjusting plate with a lock, which allows changing the sliding angle in the range from 0 to 70 degrees. A laboratory setup can be used to study the cutting process of various feeds used in animal husbandry. The analysis of the most important parameters affecting the specific work of feed cutting was carried out, the dependence of the influence of the grinder knife design parameters (blade thickness and sliding angle) on specific cutting work of straw, potatoes, feed beetroot, carrots, corn cobs, stalks and leaves was established. The results of the laboratory studies indicate that at certain (appropriate) values of the knife sliding angle of the grinder, it is possible to achieve a decrease of specific cutting work of some feed (corn cobs and stalks, potatoes, feed beetroot, carrots) by $25 \ldots 37 \%$, when cutting corn leaves - by $15 \%$, wheat straw - by $3 \%$. This dependence is typical of a wide range of cutting speeds of the materials under study (from 2 to $10 \mathrm{~m} / \mathrm{s}$ ), the differences fit into the measurement inaccuracy. The results of the laboratory studies and the presented method can be used for design development of grinder cutting device for livestock enterprises.
\end{abstract}

\section{Introduction}

Numerous studies of scientists indicate that animal feed digestibility depends mainly on feed nutrient balance, as well as on the size of feed particles. When the size of feed particles is larger than those established by the zootechnical requirements, they are not fully digested by the animal body, and when the sizes are smaller than required, the animal does not chew the food well, as a result, it is not sufficiently wetted with saliva and is partially swallowed unchewed.

In addition, feed grinding solves the problem of improving the conditions of mechanization and automation of the processes of feed mixing, dosing and distributing it to animals.

Currently, the country industry produces feed grinders that work according to long-known and outdated principles and schemes, which negatively affects the grinding energy consumption and the quality of the resulting feed, as well as the demand for mechanization equipment for a similar purpose. Great competition in the feed grinder market imposes more stringent requirements for design, energy and operational characteristics of grinders, as well as for quality parametres of the process itself.

The most widespread machines are those that use the method of grinding by cutting, since cutting, in comparison with the material destruction by impact or crushing, is the least energy intensive method. In addition, when destructive elements hit the crushed product, cell fluid is released, which contradicts zootechnical requirements.

This makes the issues of comprehensive research of the cutting process of various feeds currently important in order to improve both the process itself and the equipment intended for its realization.

The study of feed cutting process is a task with a complex structure due to a large number of factors that influence the grinding process. Factors affecting the cutting process can be divided into three groups:

- physical and mechanical properties of feed;

- design of the grinding body and characteristics of the grinder working elements;

- operation modes of the grinders.

It should also be considered that they have a complex mutual influence on the cutting process, and combinations of factors can significantly influence the nature of the interaction.

Basic parameters that influence the energy components of feed cutting process include: cutting speed, geometric parameters of the knife.

1. Cutting speed.

Cutting speed is one of the most important parameters affecting the specific work value of feed material cutting; in addition, the value of this parameter directly influences the cutting body performance and the size of the chopped feed particles.

\footnotetext{
*Corresponding author: nikall85g@yandex.ru
} 
A number of studies [1-4] devoted to identifying the suitable value of the feed cutting speed indicate that as this parameter increases in a wide range, the energy consumption for grinding decreases.

Some of the studies [5-8] indicate a decrease of specific feed cutting work only until a certain speed is reached, then the energy component of the cutting process increases.

2. Angle of sharpening.

Studies carried out by numerous scientists indicate a definite decrease of specific feed cutting work with a decrease of the knife sharpening angle (the angle formed by the knife blade bevels to its cutting edge, measured in a plane perpendicular to its edge).

Moreover, it should be noted that the dependence of the knife blade sharpening angle on specific feed cutting work usually has the form of a hyperbole. In case of sharpening angle increase from 15 to $30^{\circ}$, there is an almost linear increase of specific cutting work of most feed crops by $15 \ldots 25 \%$, depending on the type of material to be cut, the knife thickness. With a further increase of the knife sharpening angle, there is a steep increase of specific cutting work.

Recommendations on selection of the suitable sharpening angle of the feed grinder cutting body can be summarized as follows. To reduce energy consumption for feed cutting, it is necessary to ensure a minimum sharpening angle of the knives.

However, with a decrease of this parameter value, knife wear resistance and strength decreases, which negatively affects the grinder reliability. It is advisable to calculate the appropriate sharpening angle based on the requirements for strength and wear resistance of knives. It is expedient to sharpen knives within $12 \ldots$ $22^{\circ}$ for grinding most of the feeds.

3. Sliding angle.

When developing cutting devices for grinders, the utmost importance is placed to substantiation of the sliding angle of its knives. The influence of the sliding angle on cutting energy performance is generally recognized.

Ensuring the appropriate sliding angle of the cutting body knives and its scheme is an important component and it has become a criterion for assessing the knife shape rationality. Regarding the appropriate value of the sliding angle, various appropriate values of this parameter are given in the literature.

Academician V.A. Zheligovsky gives data that the lowest energy parameters are typical at a sliding angle of 0 degrees, i.e. at the chopping action of the knife, and with an increase of this parameter value, the growth of specific cutting work of plant materials accelerates.

Studies carried out by other researchers [9-11] indicate specific work decrease of cutting various feeds with a sliding angle of 25 to $50^{\circ}$ (when cutting carrots and apples $-30^{\circ}$, timothy $-35^{\circ}$, corn $-40^{\circ}$, horse meat and pollack $-50^{\circ}$ ).

Analysis of the research data shows that at the moment there are no scientifically based recommendations that could be used in design of cutting bodies for animal feed grinders.
4. The thickness of the cutting element.

Experimental studies $[12,13]$ show that with an increase of the knife thickness, specific cutting work increases. The blade thickness ranges from 5 to $15 \mathrm{~mm}$ in most cutting bodies of feed chopping machines.

Research conducted by N.I. Reznik [13], when cutting corn with the knives of 2 to $8 \mathrm{~mm}$ thick, made it possible to establish an increase of specific cutting work from 45 to $67 \%$, which is a significant value. It should also be noted that with an increase of the knife thickness from 2 to $5 \mathrm{~mm}$, there is a sharp increase of specific cutting work, a further increase of the knife blade thickness up to $8 \mathrm{~mm}$ leads to a smooth increase of specific cutting work.

5. The slot between the edges of the cutting pair blades.

The slot between the edges of the cutting pair blades is of substantive importance for specific work change of feed material cutting.

The studies reflected in the works $[13,14]$ made it possible to establish an increase of specific cutting work with an increase of the slot between the edges of the cutting pair blades.

N.E. Reznik [13] found that with an increase of the slot between the edges of the cutting pair blades from 2 to $10 \mathrm{~mm}$, the specific cutting work increases $1.4 \ldots 5$ times when cutting most of the feed materials, depending on the crop to be chopped and the geometric parameters of the knives.

\section{Materials and methods}

To study the process of feed cutting of vegetable origin, a laboratory setup based on the principle of an impact machine, which made it possible to provide a cutting speed in a sufficiently wide range, was developed at Ulyanovsk State Agrarian University (Figure 1).

The principle of the laboratory setup is as follows. The laboratory setup knife (Figure 2) is fixed on a flywheel mounted on a shaft, the torque to which is transmitted from the electric motor through a belt drive and a freewheel clutch. At the rotation frequency of the flywheel corresponding to the required cutting speed of the feed material, the electric motor is turned off through the control panel in order to exclude the inertia moment influence of the electric motor and the sample of the material is put into the knife cutting plane. For a short-term supply of the test material into the knife cutting plane, a current is momentarily applied to the solenoid winding of the stock feeder, which pushes the fixing core with the fixed test material into the cutting plane, then the fixing core returns to its primary position.

The strain-gage sensor is mounted on the fixing mechanism and allows to register the cutting force. The strain gauge signal goes to the amplifier, then to the analog-to-digital converter and is transmitted to the screen of the personal computer.

The flywheel rotation frequency after cutting the studied material sample with a knife is recorded with a 
tachometer. The work expended on a sample cutting reduces the flywheel speed.

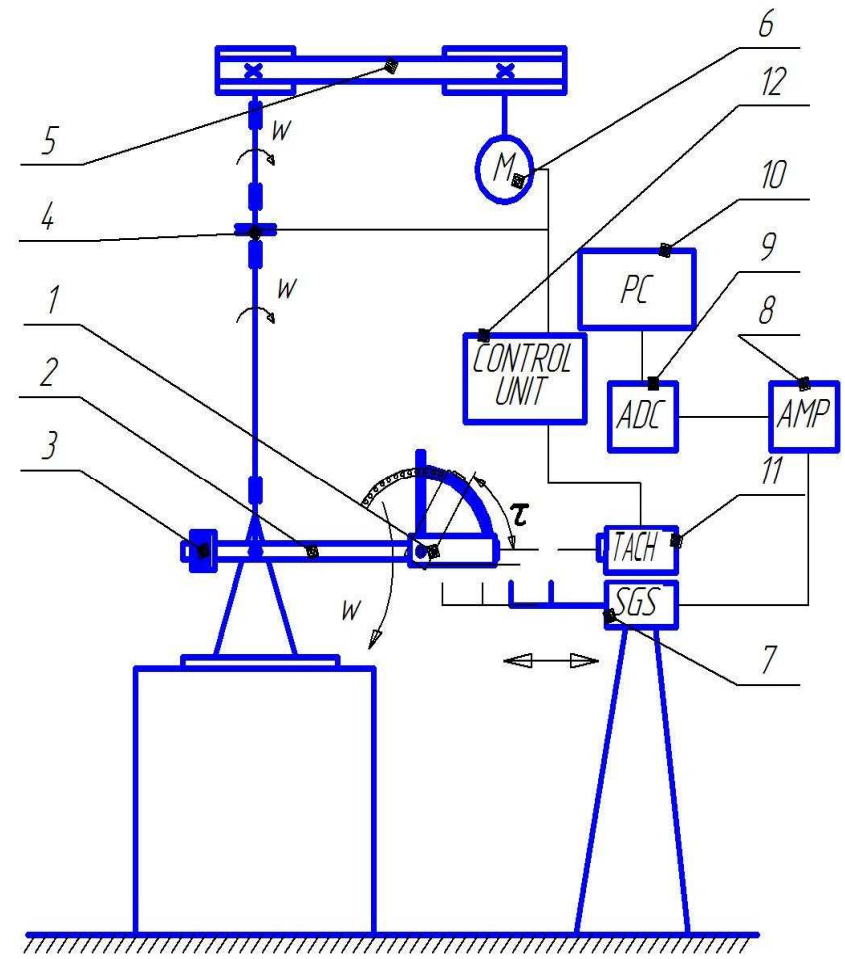

Fig. 1. Schematic illustration of a laboratory setup for studying the process of feed cutting: 1 - knife; 2 - flywheel; 3 - counterweight; 4 - freewheel clutch; 5 - belt drive; 6electric motor; 7 - stock feeder with a strain-gage sensor; 8 amplifier; 9 - analog-to-digital converter; 10 - personal computer; 11-tachometer; 12 - control unit

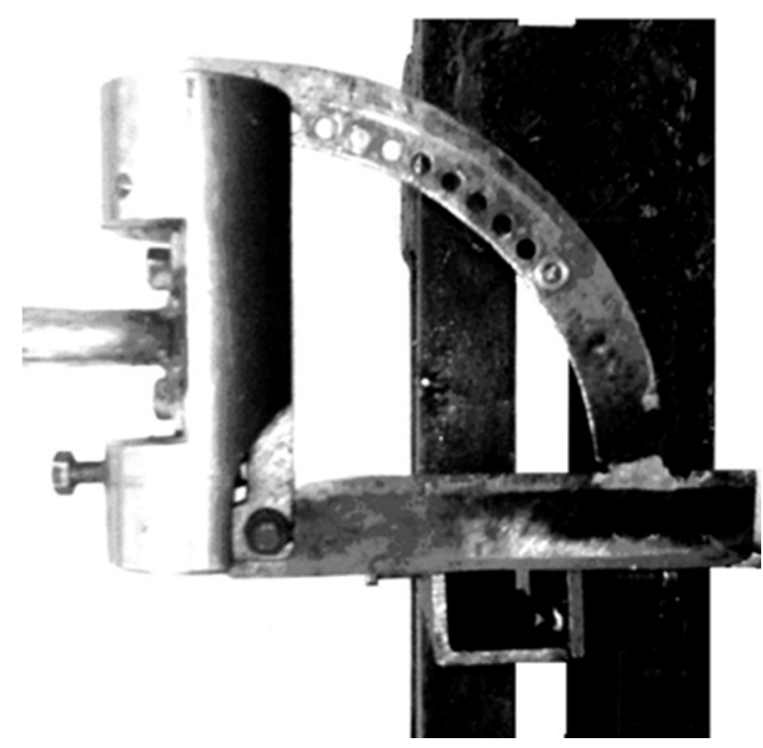

Fig. 2. Knife with the possibility to adjust the cutting sliding angle

Having the data on the flywheel rotation frequency before and after cutting, the cutting work can be calculated.

$$
A=k\left(n^{2}{ }_{1}-n^{2}{ }_{2}\right)
$$

where $\mathrm{k}$ is a coefficient depending on the polar inertia moment of all rotating parts of the laboratory setup;

$$
\mathrm{k}=2 \cdot \pi^{2}\left(\mathrm{~J}_{\mathrm{fr} . \mathrm{cl}, \mathrm{sh}}+\mathrm{J}_{\mathrm{flw}, \mathrm{kn}}\right),
$$

where $\mathbf{J}_{\text {fr.cl, sh }}, \mathbf{J}_{\mathrm{fl} w}, \mathrm{kn}$ are inertia moments of the freewheel clutch with a shaft and a flywheel with a knife, respectively, $\mathrm{kg} \cdot \mathrm{m}^{2} ; \mathrm{n}_{1}$ is the flywheel rotation frequency before the cut, $\mathrm{s}^{-1} ; \mathrm{n}_{2}$ is the flywheel rotation frequency after the cut $\mathrm{s}^{-1}$.

To determine the specific cutting work, the expression was used:

$$
\mathrm{A}_{\mathrm{sp}}=\mathrm{A} / \mathrm{S}
$$

where $\mathrm{S}$ is the sectional area of the cut sample of the test material, $\mathrm{m}^{2}$.

The flywheel speed and, as a consequence, the cutting speed is controlled by the speed of the electric motor and change belt pulley drive.

To ensure the required sliding angle, the cutting element (knife) has an adjusting plate with a lock, which allows to change the sliding angle in the range from 0 to 70 degrees.

The number of repetitions for each experiment was at least 3 (if the data were discrepant, the number of repetitions was 5 .)

Studies on the sliding angle influence on the cutting work were carried out on the stems of winter wheat straw, corn leaves, stalks and cobs, potatoes, feed beetroot and carrots.

When processing the research results, the following was used: differential and integral calculations, methods of mathematical statistics, correlationregression analysis.

The reliability of the data obtained is ensured by the use of methods of mathematical processing and statistical analysis of research results, multivariative analysis, licensed mathematical software packages for personal computers: "Statistica", "Derive" and "Microsoft Office Excel".

\section{Results and discussion}

The dependence of the specific cutting work on the sliding angle is shown in Figure 3.

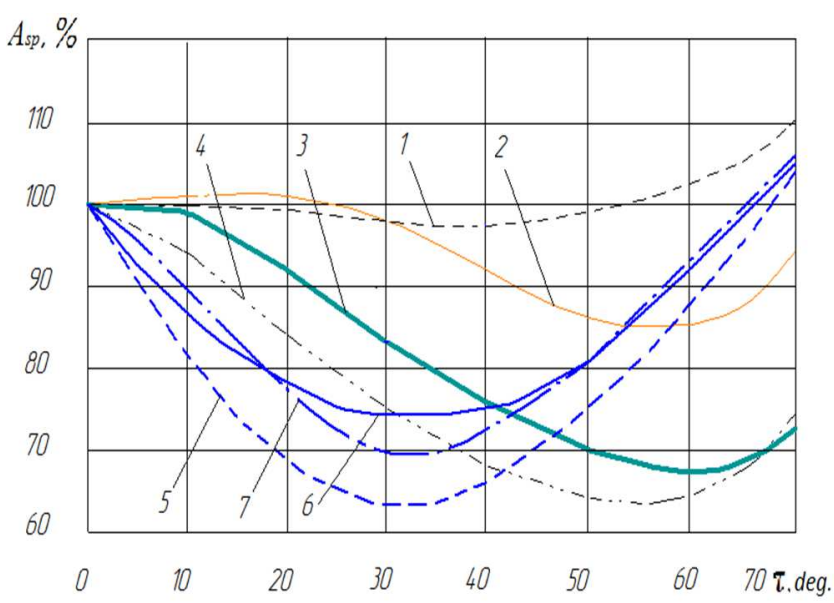

Fig. 3. Dependence of specific feed cutting work on the knife sliding angle: 1 - wheat straw; 2 - corn leaves; 3 - corn cobs; 4 - corn stalks; 5 - potatoes; 6 - feed beetroot; 7 - carrots 
The results of the study indicate that when cutting potatoes, feed beetroot and carrots, there is a decrease of specific cutting work at a sliding angle $\tau=30 \ldots 35^{\circ}$ by $37 \ldots 25 \%$ compared to $\tau=0^{\circ}$, with a further increase of the sliding angle to $70^{\circ}$ there is an increase of specific cutting work by $5 \%$.

When cutting corn, a decrease of specific cutting work occurs at a sliding angle of $55 . .65^{\circ}$, and the decrease is by $15 \ldots 37 \%$, depending on the material under study (leaves, cobs and stalks). With the sliding angle increase to $70^{\circ}$, the specific cutting work is lower than with $\tau=0^{\circ}$ by $6 \ldots 28 \%$. When cutting winter wheat straw, the specific cutting work reduction is only $3 \%$ at a sliding angle of $30 . .45^{\circ}$. With an increase of the sliding angle to $70^{\circ}$, the specific cutting work increases by $10 \%$ than at $\tau=0^{\circ}$.

This dependence is typical for a wide range of cutting speeds of the materials under study (from 2 to $10 \mathrm{~m} / \mathrm{s}$ ), the differences fit into the measurement inaccuracy.

A decrease of specific feed cutting work is associated with the transformation of the sharpening angle and is presented in the diagram (Figure 4).

The knife is with a sharpening angle $\beta$ (formed by the doc points). When the knife enters the material under study at an angle $\tau$ (sliding angle) in the direction $\mathrm{v}_{\mathrm{p}}$, the sharpening angle will be $\beta_{1}$ (formed by points aob).

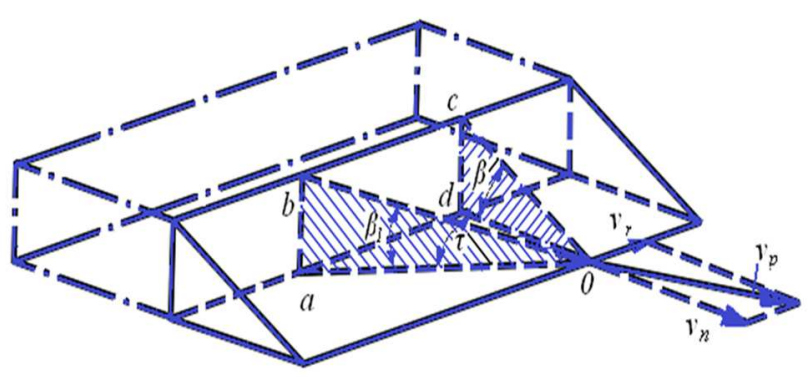

Fig. 4. Transformation of the knife sharpening angle

With executing of oblique cutting and an increase of the sliding angle $\tau$, the sharpening angle $\beta_{1}$ can decrease several times compared to the initial value $\beta$.

An increase of specific cutting work during feed grinding in case of an increase of the sliding angle is directly related to the contact area increase between the knife bevels and the material being chopped and, as a consequence, the knife friction force against the material.

The dependence of the change of the knife contact area and the chopped material has the form:

$$
\mathrm{S}_{\mathrm{a}}=\mathrm{S}_{0} / \cos \tau,
$$

where $\mathrm{Sa}$ is the actual knife contact area with the material to be chopped, $\mathrm{m}^{2} ; \mathrm{S}_{0}$ is the knife contact area with the material to be chopped at a sliding angle of zero degrees, $\mathrm{m}^{2} ; \tau$ - sliding angle, deg.

Figure 5 shows the dependence of the specific feed cutting work on the knife blade thickness.

In case of the knife thickness increase from 3 to 6 $\mathrm{mm}$, the specific cutting work increases by $40 \ldots 70 \%$, depending on the material being cut. This happens due to the fact that in case of cutting with a blade, the destructive effect is exerted to a greater extent by the edge of the knife blade, and not by its thickness. As the thickness of the knife blade increases, the intensity of the increase of specific feed cutting work decreases.

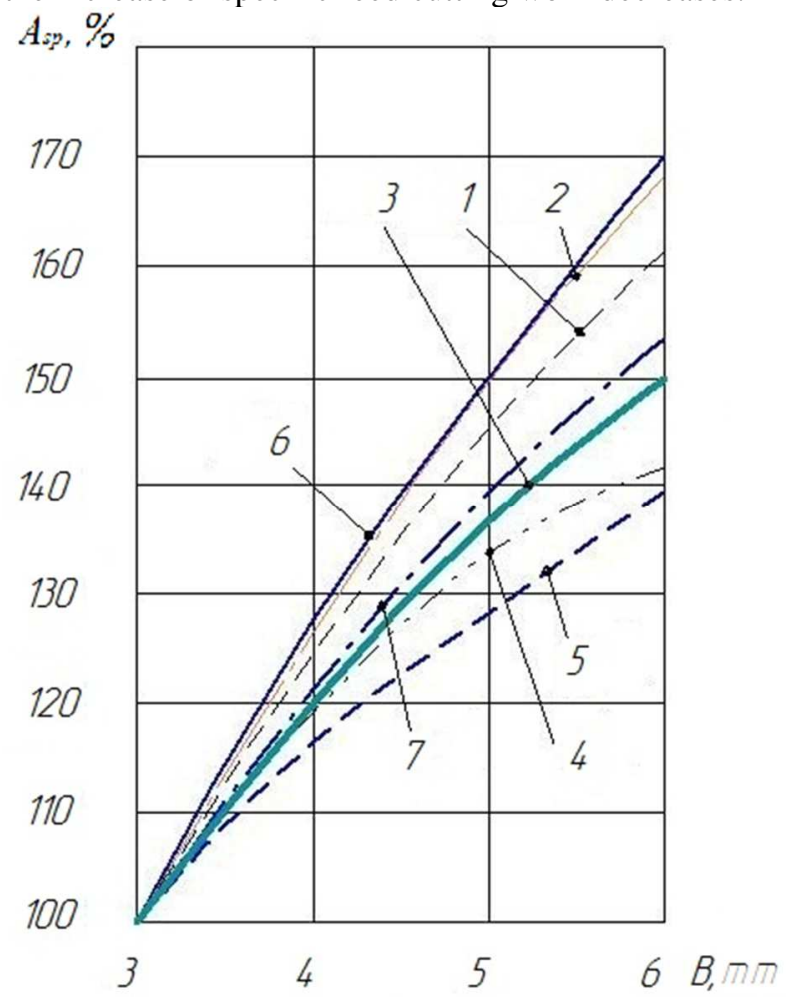

Fig. 5. Dependence of the specific feed cutting work on the knife blade thickness

\section{Conclusion}

The results of the laboratory studies indicate that at certain (appropriate) values of the knife sliding angle of the grinder, it is possible to achieve a decrease of specific cutting work of some feed (corn cobs and stalks, potatoes, feed beetroot, carrots) by $25 \ldots 37 \%$, when cutting corn leaves - by $15 \%$, wheat straw - by $3 \%$. The appropriate value of the knife sliding angle can vary in a wide range from 30 to $60^{\circ}$, which must be taken into account when developing the designs of the cutting body and the knives of the feed grinders.

Definitely, the knife blade thickness plays an important role in reducing the specific work of feed cutting, but the edge of the knife blade plays a decisive role in destructive effect of the knife on the feed material, and its thickness should be determined by the required strength characteristics.

\section{References}

1. T.A. Gavrilov, Investigation of the influence of cutting speed on the energy consumption of the process of grinding of meat raw materials, Perspective development of science, technology and technology: materials of the III International 
scientific and practical conference, 1, 263-266 (2013)

2. T.A. Gavrilov, Experimental study of the process of raw meat grinding at different cutting speeds, Scientific notes of Petrozavodsk State University, 8(137), 98-100 (2013)

3. N. Akhmetov, Research of the process of rice straw cutting and development of the working body appropriate parameters of the grinder, dissertation of the Candidate of Technical Sciences, $143 \mathrm{p}$. (Moscow, 1977)

4. N.A. Barsov, Resource-saving technological processes and technical means of processing meat and bone feed in fur farming, dissertation of the Doctor of Technical Sciences, 607 p. (Puskin, 1992)

5. V.A. Bogatov, E.I. Zotov, V.V. Khabarova, Analysis of factors determining energy consumption with vibrations during grinding of root crops and melons, Vestnik of Ulyanovsk state agricultural academy, 1(2), 67-70 (2006)

6. A.V. Burmaga, S.M. Dotsenko, L.G. Krjuchkova, V.V. Shishkin, Efficient technologies preparation of feed for pigs based on root turnip varieties kuuzik, Research Journal of Pharmaceutical, Biological and Chemical Sciences, 7(1), 11401144 (2016)

7. N.F. Baranov, Justification and improvement of the design and technological parameters of the grinder for preparation of a needle-bearing twig additive, Vestnik of Nizhny Novgorod State Engineering and Economic University, 6(61), 7-16 (2016)

8. A.A. Ivanov, Research of chopping of meat and bone raw material cutting in order to improve the relevant equipment, dissertation of the Candidate of Technical Sciences, 175 p. (Moscow, 1981)

9. V.V. Novikov, Results of production tests of an experimental root crop shredder, Izvestiya of Orenburg SAU, 2(64), 75-77 (2017)

10. V. I. Kurdyumov, Analysis of factors affecting the energy intensity of cutting, Niva Povolzhya, 3, 5759 (2008)

11. N.I. Lebed, Substantiation of design parameters and operating modes of the apple grinder, dissertation of the Candidate of Technical Sciences, 136 p. (Volgograd, 2013)

12. R Sh. Khalimov, N.P. Ayugin, L.G. Tatarov, Improvement of the feed grinder, International Research Journal, 11-4(65), 79-83 (2017)

13. N.E. Reznik, Theory of cutting with a blade and the basics of cutting device development, Mechanical engineering, 29-32 (1975)

14. J.B. Liljedahl, G.L. Jackson, R.P. De Graff, M.E. Schroeder, Measurement of shearing energy, Agricultural Engineering, 6, 298-301 (1961) 